\title{
Intervenciones en la administración de medicamentos de alto riesgo: citotóxicos
}

\author{
Interventions in the administration of high-risk drugs: cytotoxics \\ Dafne Liseth Romero Gutiérrez,* Anahí Vázquez Refugio,* \\ África Alexandra del Castillo Paulino
}

\section{RESUMEN}

El cáncer es una de las enfermedades que causa mayor mortalidad en el mundo, los tratamientos con medicamentos citotóxicos generan un sinnúmero de efectos secundarios que si no son bien atenidos podrían causar graves daños a la salud del paciente. La correcta manipulación de fármacos citotóxicos es fundamental para la seguridad del paciente; se pretende prevenir o reducir errores de medicación para mejorar la práctica de los profesionales de enfermería. Aunque las recomendaciones para la administración de estos medicamentos son muy vastas, se pretende ofrecer los siguientes puntos clave para el correcto desempeño ante una posible toxicidad inmediata.

Palabras clave: Seguridad del paciente, enfermería, preparaciones farmacéuticas, errores de medicación.

\begin{abstract}
Cancer is one of the diseases that causes the highest mortality in the world, this type of treatment generates a number of side effects that, if not well treated, it's could cause serious damage to the patient's health. The correct handling of cytotoxic drugs is essential for patient safety; the aim is to prevent or reduce medication errors in order to improve the practice of nursing professionals. Although the recommendations for administering these drugs are very broad, the intention is to offer key points, for correct action in case of possible immediate toxicity.
\end{abstract}

Keywords: Patient safety, nursing, pharmaceutical preparations, medication errors.
* Programa de Servicio Social en Investigación (SSICONAMED). Facultad de Estudios Superiores Iztacala. UNAM.

₹ Maestría en Tanatología. Instituto Mexicano de Psicooncología.

Correspondencia: DLRG, dafne.romero.g@gmail. com

Conflicto de intereses:

Los autores declaramos que no tenemos conflicto de intereses.

Citar como: Romero GDL, Vázquez RA, Del Castillo PÁA. Intervenciones en la administración de medicamentos de alto riesgo: citotóxicos. Rev CONAMED. 2020; 25(4): 193-196. https://dx.doi. org/10.35366/97340

Financiamiento: No se recibió ningún tipo de apoyo económico o financiamiento del sector público, comercial, o sin ánimo de lucro.

Recibido: 03/11/2020. Aceptado: 04/11/2020. 


\section{INTRODUCCIÓN}

Los citotóxicos son modificadores de la enfermedad cuyo mecanismo de acción se basa en provocar la muerte celular o impedir la proliferación de forma selectiva y controlada de las células y mediadores inflamatorios.' El tratamiento puede generar efectos adversos con consecuencias graves (23\% de invalidez permanente). ${ }^{2}$

Pueden causar efectos adversos como mutaciones, malformaciones congénitas, esterilidad, entre otros; el riesgo varía de acuerdo a la dosis, la frecuencia y duración de la exposición. Las personas con mayor riesgo de complicaciones por el uso de estas drogas son los pacientes que las reciben, el personal que las manipula y aquéllos que están en contacto con el material de desecho (excretas del paciente, residuos y sobrantes de la preparación).3,4

Los citostáticos más implicados en las órdenes médicas corresponden a ciclofosfamida (21\%), adriamicina (15\%), carboplatino (11\%), 5-fluoracilo (9\%), ametopterina (8\%) como los más representados, y cisplatino (5\%), vinblastina (4\%), bleomicina (3\%), dacarbacina (3\%), paclitaxel, docetaxel y vincristina.? El uso de citotóxicos en pacientes adultos mayores de 70 años puede presentar mayor riesgo de neutropenia (número reducido de neutrófilos en la sangre, generalmente menor de 1,500 o 2,000 $\mathrm{LL}$ ).' La trombocitopenia (disminución del número de plaquetas, el número normal es entre 150,000-400,000/(mm³) se reporta en 19\% de los pacientes, la cual a su vez puede incrementar el riesgo de hemorragia, la presencia de neutropenia o leucopenia (disminución de la cantidad de leucocitos, por lo general por debajo de 5,000/(mm³), aumenta el riesgo de infección.? Se han descrito algunos casos de necrosis cutánea precedida a veces por púrpura o lesiones eritematosas dolorosas ante la administración de citotóxicos?

\section{RECOMENDACIONES}

1. El profesional de enfermería involucrado en la preparación debe conocer las reglas de manejo de fármacos citotóxicos y recibir capacitación específica antes de comenzar a trabajar y de manera regular. ${ }^{5}$

2. La preparación de fármacos citotóxicos se realiza en la cabina para un paciente a la vez, y cada medicamento se prepara individualmente. ${ }^{5}$
3. El cebado de los tubos intravenosos y las jeringas se realiza dentro de la cabina de seguridad. ${ }^{5}$

4. La quimioterapia intratecal se prepara por separado de la quimioterapia intravenosa.,6

5. Existe el riesgo de salpicaduras en la administración de citotóxicos intratecales debido al aumento de las presiones que ejercen. ${ }^{5}$

6. Informar al paciente sobre los síntomas relacionados con la infusión y cuáles debe reportar de manera urgente (edema o eritema en relación con la punción venosa, dolor, escozor, rash o prurito). ${ }^{5}$

7. Efectos adversos de los citotóxicos: es importante conocer los efectos adversos para poder prevenir la aparición de toxicidad, lo que permite tener un buen control sintomático.

- Toxicidad inmediata (horas-días): náuseas, vómito, fiebre, hipertensión/hipotensión, flebitis, insuficiencia renal aguda, reacciones alérgicas, rash cutáneo, cistitis hemorrágica, necrosis tisular local.

- Toxicidad precoz (días-semanas): alopecia, leucopenia, anemia, trombopenia, mucositis, diarrea, íleo paralítico, hiperglucemia, psicosis, retención hídrica, síndrome pseudogripal.

- Toxicidad retardada (semanas-meses): ototoxicidad, anemia, aspermia, fibrosis pulmonar, neuropatía periférica, cardiotoxicidad, ataxia cerebelosa, daño hepatocelular, fenómeno de Raynaud, síndrome hemolíticourémico, hiperpigmentación cutánea.

- Toxicidad tardía (meses-años): hipogonadismo/esterilidad, leucemias agudas, linfomas, encefalopatía, cataratas, carcinogénesis, menopausia precoz, fibrosis hepática/cirrosis, osteoporosis. ${ }^{7}$

8. Muchos antineoplásicos deben protegerse de la luz.2

9. Al manipular cualquier agente citotóxico debe evitarse su inhalación o el contacto con la piel - los ojos. Si ocurre contacto, el área afectada debe lavarse con agua abundante. ${ }^{2}$

10. Las batas utilizadas para preparar y/o ministrar fármacos citotóxicos deben ser desechables y cambiarse en caso de contaminación, derrames o roturas, y al finalizar cada procedimiento. ${ }^{4}$ 
11. Debe existir la disponibilidad de un kit de manejo de derrames en todas las áreas donde se manipulan y administran los medicamentos citotóxicos. $^{4}$

12. Antes de comenzar el tratamiento con algún antineoplásico es necesario cuantificar los leucocitos (con diferencial), la hemoglobina y las plaquetas. $^{2}$

13. Monitorear la biometría hemática cada semana.?

14. Los citotóxicos deben administrarse en ayunas por lo menos una hora antes de los alimentos.'

15. La premedicación con un antiemético reduce las náuseas.' Administrar de manera independiente a través de una vía intravenosa específica, puesto que muchos antineoplásicos son incompatibles con otras sustancias. Las vías intravenosas deben purgarse después de su uso. ${ }^{2}$ No se recomienda el uso de bombas de infusión a menos que se reduzca la presión, por lo que es importante realizar un doble chequeo del medicamento, concentración, dosis, velocidad de infusión y colocación de la vía. ${ }^{6-8}$

16. Deben elegirse vasos de gran calibre en el antebrazo evitando venas con problemas vasculares así como las áreas articulares (muñeca o el pliegue del codo). ${ }^{4}$

17. Revisar cualquier prueba de infiltración cuando se administra una solución de citostáticos (palidez, edema, enlentecimiento de la velocidad de perfusión o pérdida del retorno. ${ }^{4}$

18. Evitar la extravasación de fármacos citotóxicos, asegurándose de que el acceso intravenoso se mantenga fijo en su posición, con lo que se evita el dolor intenso y el daño tisular. ${ }^{2}$

19. Si se presenta extravasación, se debe detener la infusión inmediatamente, la extremidad debe elevarse y aplicarse una compresa fría durante 45 min y notificar al médico tratante. Valorar el área constantemente, puesto que la extravasación puede ser progresiva. ${ }^{2}$

20.Se sugiere la aplicación de compresas frías para la extravasación de todos los fármacos vesicantes o irritantes, excepto los alcaloides de la vinca (vincristina, vinblastina, vinorelbina) y epipodofilotoxinas tales como etopósido. El frío intermitente causa vasoconstricción, disminuye la propagación del fármaco, la extensión de la lesión, la inflamación y el dolor. ${ }^{4}$
21. Para la extravasación de alcaloides de la vinca o epipodofilotoxinas se sugiere la aplicación de calor seco, ya que causa vasodilatación localizada y aumento del flujo sanguíneo, mejorando la eliminación del fármaco. ${ }^{4}$

22. Ante la presencia de algún efecto indeseable, disminuir la infusión y notificar al médico para su suspensión.?

23. Ante la sospecha de una sobredosis se debe interrumpir la administración de los citotóxicos?

24. La fiebre puede aparecer como efecto secundario o asociada a una infección producida por el descenso de la cifra de neutrófilos secundaria a la quimioterapia.?

25. La utilización de fármacos de platino está contraindicada en reacciones de hipersensibilidad anterior debido a la existencia de reacciones cruzadas entre ellas. El tratamiento consiste en la administración de adrenalina, corticosteroides y antihistamínicos así como en el cese de la medicación. ${ }^{9}$

26. El desecho de frasco-ámpula y equipos utilizados con citotóxicos deberá ser depositado en bolsas rojas.

\section{Biblografía}

1. Intervenciones de Enfermería para la Seguridad en la Administración de Medicamentos de Alto Riesgo: Guía de Práctica Clínica. México, CENETEC; 2014 [21 Enero de 2020]. Disponible en: http://www.cenetec-difusion.com/ CMGPC/IMSS-712-14/ER.pdf.

2. Tiziani A. Havard: fármacos en enfermería. México D.F.: Manual Moderno; 2011.

3. Coynea E, Northfieldb S, Ashc K, Brown-Westd L. Current evidence of education and safety requirements for the nursing administration of chemotherapy: an integrative review. Eur ] Oncol Nurs. [Internet] 2019 [Cited 2020 September 03]; 44: 24-32. Available from: https://wwwclinicalkey-es.pbidi.unam.mx:2443/service/content/pdf/ watermarked/1-s2.0-S1462388919300493.pdf?locale=es_ ES\&searchlndex=.

4. Kohen N, Viniegra M. INC. Procedimientos básicos para la preparación, administración y desecho de drogas citostáticas. INC. [Internet] 2016 [Citado 2020 Agosto 24] Disponible en: 0000000892cnt-2016-10-11-documentoprocedimiento-de-manejo-de drogas-citostáticas2016pdf.

5. Vera R, Otero MJ, Ayala de la Peña F. Recommendations by the Spanish Society of Hospital Pharmacy, the Spanish Society of Oncology Nursing and the Spanish 
Society of Medical Oncology for the safe management of antineoplastic medication in cancer patients. Farm Hosp [Internet] 2018 [Cited 202001 September]; 42 (6): 261-268. Available from: https://pubmed.ncbi.nlm.nih. gov/30298467/.

6. Otero LM. Prácticas para mejorar la seguridad de los medicamentos de alto riesgo. [Internet]. Plan de Calidad para el Sistema Nacional de Salud. 2007 [Citado 2020 Enero 27]. Disponible en: http://www.ismp-espana.org/ ficheros/Practicas para mejorar la seguridad de los medicamentos de alto riesgo.pdf.

7. Blasco A, Caballero C. Toxicidad de los tratamientos oncológicos SEOM [Internet] España. [Citado 2020 Septiembre 01]. Disponible en: https://seom.org/ guia-actualizada-de-tratamientos/toxicidad-de-lostratamientos-oncologicos?showall=1.
8. Magallón Pedrera I, Pérez Altozano J, Virizuela Echaburu AJ, Beato Zambrano C. Borrega García P, de la Torre Montero JC. Safety recommendations guideline for cancer patients receiving intravenous therapy. ECO-SEOM-SEEO [Internet] 2020 [Cited 2020 August 26]. Available from: https://seom. org/images/SEOM_Safety_recommendations_guideline_ cancer.pdf.

9. Sanmartína O, Beato C, Jin Suh-Ohc H, Aragón I, España A, Majemf $M$ et al. Clinical management of cutaneous adverse events in patients on chemotherapy: a national consensus statement by the Spanish Academy of Dermatology and Venereology and The Spanish Society of Medical Oncology. Actas Demo-Sifiliograficas [Internet] 2019. [Cited 2020 Sept. 01]; 170 (6): 448-459. Available from: https://www-clinicalkeyes.pbidi.unam.mx:2443/service/content/pdf/watermarked//s2.0-S1578219019301507.pdf?locale=es_ES\&searchIndex=. 